\title{
SURFACES WITH SIMPLE GEODESICS
}

\author{
By \\ Yury NiKolayevsKY
}

\begin{abstract}
A regular submanifold in a Euclidean space $\boldsymbol{R}^{N}$ is called a submanifold with simple geodesics if all its geodesics have constant Frenet curvatures in $\boldsymbol{R}^{N}$. A submanifold with congruent simple geodesics is called helical. We prove that a compact surface with simple geodesics is either a rational torus, or the image of the unit sphere $S^{2}(1) \subset \boldsymbol{R}^{3}$ under a polynomial map $F: \boldsymbol{R}^{3} \rightarrow \boldsymbol{R}^{N}$ of the special structure. As a corollary, a compact surface $F^{2} \subset \boldsymbol{R}^{N}$ is helical if $F^{2}=\Phi\left(S^{2}\right)$, where $\Phi=\left(a_{1} \Phi_{1}, \ldots, a_{m} \Phi_{m}\right)$ and $\Phi_{i}$ the $i$-th eigenmap of the Laplacian of $S^{2}$.
\end{abstract}

\section{Introduction}

The main object of our study is surfaces with simple geodesics, that is, with geodesics of constant Frenet curvatures. Although the hypothesis of having simple geodesics proves to be rather tight in low codimensions, it does not appear so restrictive just on its own. While imposing very strong conditions on the intrinsic geometry of the surface (we will see that a compact surface with simple geodesics has constant non-negative Gauss curvature), this hypothesis leaves plenty of freedom for its shape. In fact, it appears that surfaces with simple geodesics in a Euclidean space of dimension $N$ are parametrized by the points of a cone with a nonempty interior in the space of polynomials of degree $O(\sqrt{N})$ in three real variables. By contrast, helical surfaces, surfaces with congruent simple geodesics, are quite rigid and can be constructed in a uniform way using spherical functions [13].

1991 Mathematics Subject Classification. Primary: 53A05, 53C40; Secondary: 53C22, 53A04.

Key words and phrases. simple geodesics, helical surface.

Received May 24, 1999

Revised December 13, 1999 
Definition 1 [9]. A regular curve in a Euclidean space is called a $W$-curve if all its Frenet curvatures are constant.

A $W$-curve $\gamma \subset \boldsymbol{R}^{N}$ can be written in the form:

$$
\gamma(t)=a+b t+\sum_{i=1}^{d}\left(e_{i} \cos \left(\mu_{i} t\right)+f_{i} \sin \left(\mu_{i} t\right)\right),
$$

where $t$ is an arclength (or an affine) parameter, $0<\mu_{1}<\cdots<\mu_{d}$, and $b, e_{1}, \ldots$, $e_{d}, f_{1}, \ldots, f_{d}$ are orthogonal vectors in $\boldsymbol{R}^{N}$ satisfying $\left\|e_{i}\right\|=\left\|f_{i}\right\| \neq 0$ for all $i=1, \ldots, d$. The number $d$ is called the order of the $W$-curve $\gamma$.

We will equally utilise another, more geometrically transparent, definition of $W$-curves which makes no use of Frenet curvatures and higher order derivatives:

Definition $1^{\prime}$. A regular curve $\gamma$ in a Euclidean space is called a $W$-curve if the length of a chord of $\gamma$ is a function of the length of the arc with the same endpoints.

As we see from (1), the function in Definition $1^{\prime}$ is a quasipolynomial with coefficients determined by Frenet curvatures of $\gamma$. The Frenet curvatures, in turn, can be restored from this function.

In 1982 D. Ferus, S. Schirrmacher [6] and K. Sakamoto [11] introduced the following notions:

Definition 2. A regular submanifold $F \subset \boldsymbol{R}^{N}$ is called a submanifold with simple geodesics (shortly, SG-submanifold) if all the geodesics of $F$ are $W$-curves. If, in addition, all of them are congruent, the submanifold is called helical.

An isometric immersion of a Riemannian manifold is called an immersion with simple geodesics (helical immersion), if its image has simple geodesics (respectively, is helical).

EXAMPLE 1. Among the surfaces in $\boldsymbol{R}^{3}$, only planes, spheres and circular cylinders are SG-surfaces. Spheres and planes are helical, and cylinders are not. Compact SG-surfaces in $\boldsymbol{R}^{4}$ and $\boldsymbol{R}^{5}$ are classified in [6,8]. Besides those that lie in some $\boldsymbol{R}^{3}$, there is a Clifford torus in $\boldsymbol{R}^{4}$ and a Veronese surface in $\boldsymbol{R}^{5}$.

A class of minimal nonhelical SG-immersions of $S^{3}$ to $S^{24}$ was studied in [10]. 
EXAMPLE 2. Let $F_{1} \subset \boldsymbol{R}^{N_{1}}, F_{2} \subset \boldsymbol{R}^{N_{2}}$ be two SG-submanifolds. Then their Cartesian product $F_{1} \times F_{2} \subset \boldsymbol{R}^{N_{1}+N_{2}}$ is also an SG-submanifold. A totally geodesic submanifold of an SG-submanifold is an SG-submanifold itself. Combining these observations, we can construct new SG-immersions from the old ones as follows: suppose $f_{i}: M \rightarrow \boldsymbol{R}^{N_{i}}, i=1, \ldots, p$ are isometric SG-immersions of a Riemannian manifold $M$. Then the immersion $f=\left(a_{1} f_{1}, \ldots, a_{p} f_{p}\right): M \rightarrow \boldsymbol{R}^{N}$ with $\left\{a_{i}\right\}$ constants satisfying $\sum_{i=1}^{p} a_{i}^{2}=1$ and $N=\sum_{i=1}^{p} N_{i}$ is also an isometric SG-immersion.

Another example of this sort can be constructed as follows: let $T^{n}$ be a Clifford torus defined by an immersion $f: \boldsymbol{R}^{n} \rightarrow \boldsymbol{R}^{2 n}, f\left(x_{1}, \ldots, x_{n}\right)=$ $\left(r_{1} \cos x_{1}, r_{1} \sin x_{1}, \ldots, r_{n} \cos x_{n}, r_{n} \sin x_{n}\right), r_{i}>0$ for $1 \leq i \leq n$. Choose a linear subspace $L \subset \boldsymbol{R}^{n}$. Then $f(L) \subset \boldsymbol{R}^{2 n}$ is an SG-submanifold. It is compact if and only if $L$ has a basis of rational vectors. In this case, we call $f(L)$ a rational torus.

EXAMPLE 3. The special orthogonal group $S O(n)$ is a submanifold with simple geodesics in the Euclidean space of $n \times n$-matrices. Since any (simply connected) symmetric space of compact type and a flat torus can be immersed as totally geodesic submanifolds in $S O(n)$ for sufficiently large $n$, we get an SG-immersion of any symmetric space of non-negative curvature. We know no examples of SG-submanifolds that are not locally homogeneous, as well as no examples of SG-submanifolds of negative curvature. It seems natural to conjecture that a Riemannian manifold admitting an immersion with simple geodesics is homogeneous and of non-negative sectional curvature. Note that the part of this conjecture relating to the curvature sign fails to be true in the Hilbert space, even for helical submanifolds. The Bieberbach isometric embedding of the unit $\operatorname{disc}\{z \in C:|z|<1\}$ with hyperbolic metric in $L^{2}$ defined by $x_{2 m-1}=$ $\operatorname{Re}\left(z^{m}\right) / \sqrt{m}, x_{2 m}=\operatorname{Im}\left(z^{m}\right) / \sqrt{m} m=1,2, \ldots$ is equivariant and therefore helical [2]. Another example comes from the theory of harmonic manifolds: by [12] a non-compact harmonic manifold can be helically immersed in the Hilbert space. The Damek-Ricci spaces [5] immersed in $L^{2}$ in this way give an infinite family of helical submanifolds in $L^{2}$, with sectional curvature taking negative values.

EXAMPLE 4. Let $\lambda_{k}=-k(k+1), k=0,1, \ldots$ be the eigenvalues of the Laplacian on the sphere $S^{2}$ and $\left\{V_{k}\right\}$ the corresponding eigenspaces. Then $\operatorname{dim} V_{k}=2 k+1$ and $V_{k}$ is the space of homogeneous harmonic polynomials of degree $k$ in the space $\boldsymbol{R}^{3}$ restricted to the unit sphere $S^{2} \subset \boldsymbol{R}^{3}$. Explicitly, $V_{k}$ is spanned by the functions $\operatorname{Re}\left(\left(c_{1} x_{1}+c_{2} x_{2}+c_{3} x_{3}\right)^{k}\right)_{\mid S^{2}}$, where $c=\left(c_{1}, c_{2}, c_{3}\right)$ runs 
over the set of isotropic vectors in $\boldsymbol{C}^{3}$ [7]. Let $\left(\phi_{k}^{1}, \ldots, \phi_{k}^{2 k+1}\right)$ be an $L^{2}\left(S^{2}\right)$ orthonormal basis in $V_{k}$. Then the eigenmap $\Phi_{k}=\left(\phi_{k}^{1}, \ldots, \phi_{k}^{2 k+1}\right): S^{2} \rightarrow \boldsymbol{R}^{2 k+1}$ is a homothetic immersion of the sphere with simple geodesics $[1,11]$. Using the construction from the example 2 we get an immersion $\Phi=\left(a_{1} \Phi_{1}, \ldots, a_{p} \Phi_{p}\right)$ : $S^{2} \rightarrow \boldsymbol{R}^{N}$ which has simple geodesics and is isometric under an appropriate normalization of the constants $\left\{a_{k}\right\}$. What is more, the immersion $\Phi$ is helical, since it satisfies the equation

$$
\|\Phi(x)-\Phi(y)\|=f(d(x, y))
$$

for any two unit vectors $x, y \in \boldsymbol{R}^{3}$, where $d$ is the spherical distance. The results of [13] show that all the helical immersions of CROSS's are of this form.

For an SG-immersion of the sphere, the distance $\|\Phi(x)-\Phi(y)\|, x, y \in$ $S^{2}(1) \subset \boldsymbol{R}^{3}$ may depend also on a geodesic, that is, both on the length and on the direction of the vector $x \times y$, where $\times$ is the cross-product in $\boldsymbol{R}^{3}$.

This justifies the following definition:

Definition 3. A polynomial map $F: \boldsymbol{R}^{3} \rightarrow \boldsymbol{R}^{N}$ is called an $S G$-map, if it is an orthogonal sum of two maps $F_{e}: \boldsymbol{R}^{3} \rightarrow \boldsymbol{R}^{N_{1}}, F_{o}: \boldsymbol{R}^{3} \rightarrow \boldsymbol{R}^{N_{2}}, F=F_{e} \oplus F_{o}$ : $\boldsymbol{R}^{3} \rightarrow \boldsymbol{R}^{N_{1}} \oplus \boldsymbol{R}^{N_{2}}=\boldsymbol{R}^{N}$ such that

1. $F_{e}$ is an even polynomial map satisfying

$$
\left\|F_{e}(x)-F_{e}(y)\right\|^{2}=P(x \times y)
$$

for any unit vectors $x, y \in \boldsymbol{R}^{3}$, with $P$ an even polynomial in $\boldsymbol{R}^{3}, P(0)=0$.

2. $F_{o}$ is an odd polynomial map satisfying

$$
\left\langle F_{o}(x), F_{o}(y)\right\rangle=R(x, y)=\sum_{i=1}^{m}\langle x, y\rangle^{2 i-1} S_{2 m-2 i}(x \times y),
$$

for any vectors $x, y \in \boldsymbol{R}^{3}$, with $S_{2 m-2 i}(i=1, \ldots, m)$ even homogeneous polynomials, $\operatorname{deg} S_{2 m-2 i}=2 m-2 i$.

3. The map $F$ is homothetic on the unit sphere $S^{2}(1) \subset \boldsymbol{R}^{3}$.

An SG-map takes the unit sphere in $\boldsymbol{R}^{3}$ to a surface with simple geodesics, but in general, non-helical. Indeed, let $e$ be a unit vector in $\boldsymbol{R}^{3}$ and $c$ a great circle on $S^{2}$ dual to $e$. Take two orthogonal vectors $e_{1}, e_{2} \in c$. Then by (3), (4) for any two points $u=\cos t e_{1}+\sin t e_{2}, v=\cos s e_{1}+\sin s e_{2}$ on $c$, the length of the segment $F(u) F(v)$ depends only on $d(u, v)=\arccos \cos (t-s)$. So, the image of 
every great circle of $S^{2}$ is a $W$-curve in $\boldsymbol{R}^{N}$, and these $W$-curves may be noncongruent for different circles (see Example 5 below).

In Section 2, we give an explicit construction of SG-maps. It appears that they stand in one-to-one correspondence with the points of a cone with a nonempty interior in the proper space of polynomials in three variables. The helical immersions of a sphere constitute a set of positive codimension among immersions with simple geodesics.

Our main result is the following theorem:

THEOREM. Let $F^{2} \subset \boldsymbol{R}^{N}$ be a compact surface with simple geodesics. Then either

(1) $F^{2}$ is a flat rational torus, or

(2) $F^{2}$ is the image of the unit sphere in $\boldsymbol{R}^{3}$ under an SG-map.

For helical surfaces we obtain:

Proposition. A compact helical surface is the image of a sphere under the map $\Phi=\left(a_{1} \Phi_{1}, \ldots, a_{p} \Phi_{p}\right): S^{2} \rightarrow \boldsymbol{R}^{N}$, where $\Phi_{k}$ is the $k$-th eigenmap of the Laplacian of $S^{2}$.

This follows directly from the Lemma in Section 3 and the result of [13].

Throughout the paper, we use $\langle$,$\rangle , \|\|$ and $\times$ for the Euclidean inner product, Euclidean length and the cross-product in a three-space regardless of the number field. Complex vectors of zero Euclidean length are called isotropic. We also denote $d A$ the area element on the unit sphere $S^{2}(1) \subset \boldsymbol{R}^{3}$; and $\mathscr{P}_{k}$ the space of homogeneous polynomials of degree $k$ in $\boldsymbol{R}^{3}$. All the objects (surfaces, maps, manifolds) are smooth enough (say $C^{\infty}$ ).

\section{Simple Geodesics Maps}

In this Section, we give an algorithm for constructing SG-maps. Consider separately the maps of even and of odd degree.

\section{Even maps.}

To construct a polynomial $F_{e}$ of a given degree $2 n$, we start with the map $\Psi=\left(\Psi_{2}, \ldots, \Psi_{2 n}\right): \boldsymbol{R}^{3} \rightarrow \boldsymbol{R}^{N}, N=2 n^{2}+3 n$, where $\Psi_{k}: \boldsymbol{R}^{3} \rightarrow \boldsymbol{R}^{2 k+1}$ is a vector function of homogeneous harmonic polynomials of degree $k$ that are orthonormal in $L^{2}\left(S^{2}\right)$. The restriction of the map $\Psi_{k}$ to the unit sphere is $\Phi_{k}$, the $k$-th 
eigenmap of the Laplacian of $S^{2}$. The surface $\Psi\left(S^{2}\right)$ is helical and we have

$$
\|\Psi(x)-\Psi(y)\|^{2}=G(x \times y)=\sum_{k=1}^{n} c_{k}\|x \times y\|^{2 k}
$$

for unit vectors $x, y$ and some constants $c_{1}, \ldots, c_{n}$. Moreover, the map $\Psi$ is masssymmetric, that is, $\int_{S^{2}} \Psi(x) d A(x)=0$.

Now pick an arbitrary polynomial $H(z) \in \mathscr{P}_{0} \oplus \mathscr{P}_{2} \oplus \cdots \oplus \mathscr{P}_{2 n}$ and set

$$
P(z)=H(z)+A G(z)=\sum_{i=1}^{n} P_{2 i}(z), \quad P_{2 i} \in \mathscr{P}_{2 i},
$$

the constant $A$ to be determined later.

Find a map $F_{e}$ satisfying (3) with the polynomial $P$. After a parallel translation we may always assume $F_{e}$ to be mass-symmetric. Averaging (3) over the unit sphere $\|y\|=1$ gives $\left\|F_{e}(x)\right\|^{2}+C=Q(x)$, where

$$
Q(x)=\frac{1}{4 \pi} \int_{S^{2}} P(x \times y) d A(y)=\sum_{i=0}^{n} Q_{2 i}(x), \quad Q_{2 i} \in \mathscr{P}_{2 i},
$$

and $C=(4 \pi)^{-1} \int_{S^{2}}\left\|F_{e}(y)\right\|^{2} d A(y)$. Averaging once again over the unit sphere $\|x\|=1$, we find $C=(8 \pi)^{-1} \int_{S^{2}} Q(x) d A(x)$. From (3) $\left\langle F_{e}(x), F_{e}(y)\right\rangle=$ $-(P(x \times y)+2 C-Q(x)-Q(y)) / 2$ for any two unit vectors $x, y \in \boldsymbol{R}^{3}$. Now set $\tilde{P}=\tilde{P}(x, y)$ to be a homogeneous polynomial of degree $2 n$ in $x$ and of degree $2 n$ in $y$ that coincides with the polynomial $-(P(x \times y)+2 C-Q(x)-Q(y)) / 2$ on the product of spheres $S^{2} \times S^{2}=\left\{(x, y) \in \boldsymbol{R}^{6}:\|x\|=\|y\|=1\right\}$, that is,

$$
\begin{aligned}
\tilde{P}(x, y)=-\frac{1}{2}( & \sum_{i=1}^{n} P_{2 i}(x \times y)\|x\|^{2 n-2 i}\|y\|^{2 n-2 i}+C\|x\|^{2 n}\|y\|^{2 n} \\
& \left.-\|y\|^{2 n} \sum_{i=0}^{n} Q_{2 i}(x)\|x\|^{2 n-2 i}-\|x\|^{2 n} \sum_{i=0}^{n} Q_{2 i}(y)\|y\|^{2 n-2 i}\right) .
\end{aligned}
$$

The polynomial $\tilde{P}$ is a symmetric bilinear form in the space $\mathscr{P}_{2 n}^{0}$ of masssymmetric homogeneous polynomials of degree $2 n$. Choose a basis $\psi(x)=$ $\left(\psi_{1}(x), \ldots, \psi_{N}(x)\right)$ in $\mathscr{P}_{2 n}^{0}$ and define a symmetric $N \times N$ matrix $\mathscr{S}(P)$ by the equation $\tilde{P}(x, y)=\sum_{\alpha, \beta=1}^{N} \mathscr{S}(P)_{\alpha \beta} \psi_{\alpha}(x) \psi_{\beta}(y)$. The matrix $\mathscr{S}(P)$ depends linearly on $P$ and is positive definite for $P=G$ (in fact, $\mathscr{S}(G)$ is an identical matrix in the basis $\psi$ of coordinate functions of maps $\left.\|x\|^{2 n-2 k} \Psi_{2 k}(x) k=1, \ldots, n\right)$. So, picking a large enough constant $A$, we assure the non-negativeness of the matrix $\mathscr{S}(P)$. Let $\mathscr{A}$ be a square root of $\mathscr{S}(P)$, that is, $\mathscr{S}(P)={ }^{t} \mathscr{A} \mathscr{A}$. Then the polynomial 
map

$$
F_{e}(x)=\mathscr{A} \psi(x)
$$

satisfies (3) for the given polynomial $P$ on the right-hand side.

Conversely, every map $F_{e}$ respecting (3) can be obtained in this way. So, the set $\left\{F_{e}, \operatorname{deg} F_{e}=2 n\right\}$ is in a one-to-one correspondence with the points of a cone with non-empty interior in the space $\mathscr{P}_{0} \oplus \mathscr{P}_{2} \oplus \cdots \oplus \mathscr{P}_{2 n}$.

\section{Odd maps.}

The construction of the map $F_{o}$ is similar to that for the map $F_{e}$. To find the map $F_{o}$ of degree $2 m-1$ rewrite the right-hand side of (4) in the form

$$
R(x, y)=S(x, y)+B\langle x, y\rangle^{2 m-1}, \quad S(x, y)=\sum_{i=1}^{m-1}\langle x, y\rangle^{2 i-1} S_{2 m-2 i}(x \times y)
$$

for $x, y \in \boldsymbol{R}^{3}$, with $B$ the constant to be determined later.

The polynomial $R(x, y)$ is a symmetric bilinear form in the space $\mathscr{P}_{2 m-1}$. Let $\mathscr{T}(R)$ be its matrix in some basis $\sigma(x)=\left(\sigma_{1}(x), \ldots, \sigma_{M}(x)\right)$ in $\mathscr{P}_{2 m-1}$, $\left(M=\operatorname{dim} \mathscr{P}_{2 m-1}=2 m^{2}+m\right)$. Then $\mathscr{T}(R)=\mathscr{T}(S)+B \mathscr{T}\left(\langle x, y\rangle^{2 m-1}\right)$. Since the matrix $\mathscr{T}\left(\langle x, y\rangle^{2 m-1}\right)$ is positive definite (it is diagonal with positive elements on the diagonal in the basis of monomials in $\left.\mathscr{P}_{2 m-1}\right)$, we can make $\mathscr{T}(R)$ nonnegative taking the constant $B$ large enough. Find a matrix $\mathscr{B}$ such that $\mathscr{T}(R)={ }^{t} \mathscr{B} \mathscr{B}$. Then the polynomial map

$$
F_{o}(x)=\mathscr{B} \sigma(x)
$$

satisfies (4) for the given polynomial $R$ on the right-hand side.

Conversely, every map $F_{o}$ respecting (4) can be obtained in this way and the set $\left\{F_{o}, \operatorname{deg} F_{o}=2 m-1\right\}$ is in a one-to-one correspondence with the points of a cone with non-empty interior in the space $\mathscr{P}_{0} \oplus \mathscr{P}_{2} \oplus \cdots \oplus \mathscr{P}_{2 m-2}$.

The map $F_{o}$ takes the unit sphere into a sphere in $\boldsymbol{R}^{M}$, and maps antipodal points to antipodal. In contrast, the image of the unit sphere under $F_{e}$ does not in general lie in any sphere in $\boldsymbol{R}^{N}$, although $F_{e}$ is mass-symmetric, and the antipodal points are mapped to the same point, that is, the resulting surface is an immersed projective plane.

To complete the construction of an SG-map, we need the map $F=F_{e} \oplus F_{o}$ : $\boldsymbol{R}^{3} \rightarrow \boldsymbol{R}^{N+M}$ to be homothetic on the unit sphere. Take orthonormal vectors $x, y \in \boldsymbol{R}^{3}$ and set $z=x \times y$. Then by (3), (4) for small $t$

$$
\|F(x)-F(x \cos t+y \sin t)\|^{2}=\left(P_{2}(z)+\left((2 m-1) B-2 S_{2}(z)\right) t^{2}+o\left(t^{2}\right) .\right.
$$


The map $F$ is homothetic if, and only if, the quadratic polynomial on the right-hand side is constant on the unit sphere $\{\|z\|=1\}$. This can be easily achieved by taking $P_{2}(z)=2 S_{2}(z)+C\|z\|^{2}$ with some constant $C$. Note that the map $F$ remains homothetic when we change the constants $A$ and $B$.

EXAMPLE 5. Take $P(z)=12 z_{1}^{2}+12 z_{2}^{2}, \quad R(x, y)=4\langle x, y\rangle^{3}-6\langle x, y\rangle \times$ $\left(x_{1} y_{2}-x_{2} y_{1}\right)^{2}$. The SG-map $F: \boldsymbol{R}^{3} \rightarrow \boldsymbol{R}^{13}$ constructed on these polynomials is of the form

$$
\begin{aligned}
F\left(x_{1}, x_{2}, x_{3}\right)= & \left(2 x_{1}^{3}-3 x_{1} x_{2}^{2}, \sqrt{15} x_{1} x_{2}^{2}, 2 \sqrt{3} x_{1} x_{3}^{2},\right. \\
& 2 x_{2}^{3}-3 x_{2} x_{1}^{2}, \sqrt{15} x_{2} x_{1}^{2}, 2 \sqrt{3} x_{2} x_{3}^{2}, \\
& 2 x_{3}^{3}, 3 x_{3}\left(x_{1}^{2}-x_{2}^{2}\right), \sqrt{3} x_{3}\left(x_{1}^{2}+x_{2}^{2}\right), 6 x_{1} x_{2} x_{3}, \\
& \left.\frac{2}{\sqrt{3}}\left(x_{1}^{2}+x_{2}^{2}-2 x_{3}^{2}\right), 3 \sqrt{2} x_{1} x_{3}, 3 \sqrt{2} x_{2} x_{3}\right) .
\end{aligned}
$$

The image of the unit sphere $x_{1}^{2}+x_{2}^{2}+x_{3}^{2}=1$ under the map $F$ is a non-helical surface with simple geodesics of order not greater than three which is isometric to the sphere of radius $2 \sqrt{6}$.

\section{Proof of the Theorem}

Let $F^{2} \subset \boldsymbol{R}^{N}$ be a compact surface with simple geodesics, and let $M^{2}$ be its underlying Riemannian manifold, that is, $F^{2}=f\left(M^{2}\right), f: M^{2} \rightarrow R^{N}$ an isometric immersion. Denote $U M$ the unit tangent bundle of $M^{2}$.

Every geodesic $\gamma \subset F^{2}$ is of the form (1) with the linear term $b t$ vanishing because of compactness. Let $\boldsymbol{L}$ be a rational linear span of the set $\left\{\mu_{1}, \ldots, \mu_{d}\right\}$ and $s=\operatorname{dim}_{Q} \boldsymbol{L}$. Let $\left\{v_{1}, \ldots, v_{s}\right\}$ be a basis in $\boldsymbol{L}$. Then $\mu_{i}=\sum_{j=1}^{s} W_{i j} v_{j}, 1 \leq i \leq d$ with $W_{i j}$ forming a rational $d \times s$ matrix of rank $s$. Consider a Clifford torus $T^{d} \subset \boldsymbol{R}^{N}$ defined by an immersion $f: \boldsymbol{R}^{d} \rightarrow \boldsymbol{R}^{N}$,

$$
f\left(x_{1}, \ldots, x_{d}\right)=a+\sum_{i=1}^{d}\left(e_{i} \cos \left(x_{i}\right)+f_{i} \sin \left(x_{i}\right)\right),
$$

with the vectors $a, e_{i}, f_{i}$ as in (1). Then the closure $\bar{\gamma}$ of the geodesic $\gamma$ is a flat rational torus $f(L)$, of dimension $s$, where $L$ is a $\left(\boldsymbol{R}\right.$-)linear subspace in $\boldsymbol{R}^{d}$ spanned by the columns of the matrix $W$ (as in Example 2). Since the surface $F^{2}$ is compact, the case $s>2$ is not possible. If $s=2$, then the surface $F^{2}$ is a flat rational torus. 
In the remainder of the proof we consider the case $s=1$, that is, all the $\mu_{i}$ 's are rationally dependent for every geodesic $\gamma \subset F^{2}$. Then all the geodesics are closed and therefore have the same period [1]. Without loss of generality, suppose the common period to be $2 \pi$. Then all the frequences $\mu_{i}$ 's are integer, and the parametric equation of any geodesic $\gamma \subset F^{2}$ is of the form

$$
\gamma(t)=a+\sum_{k=1}^{n}\left(e_{k} \cos (k t)+f_{k} \sin (k t)\right)
$$

where $e_{1}, f_{1}, \ldots, e_{n}, f_{n}$ are orthogonal vectors in $\boldsymbol{R}^{N}$ with $\left\|e_{k}\right\|=\left\|f_{k}\right\|=r_{k}$, $k=1, \ldots, n$ and $r_{n} \neq 0$, although some of $r_{k}$ 's with $k<n$ may vanish. Call the number $n$ the degree of the geodesic $\gamma, n=\operatorname{deg} \gamma$. Similarly, define the degree of $a$ unit vector $p=(x, u) \in U M$ as the degree of the geodesic $\gamma(t)=f\left(\exp _{x}(t u)\right)$. The Fourrier coefficients

$$
a(p)=\int_{0}^{2 \pi} \gamma(t) d t, \quad e_{k}(p)=\int_{0}^{2 \pi} \gamma(t) \cos (k t) d t, \quad f_{k}(p)=\int_{0}^{2 \pi} \gamma(t) \sin (k t) d t
$$

are smooth vector functions on $U M$ for $k=1,2, \ldots$ and so are $r_{k}=\left\|e_{k}\right\|$ outside the zero set of $e_{k}$. Let $D \subset U M$ be the set of continuity of the function deg: $U M \rightarrow N$, that is, $p \in D$ if $\operatorname{deg} p=\operatorname{deg} q$ for all $q \in U M$ in a neighbourhood of $p$. The set $D$ is open and dense in $U M$. Indeed, suppose $U \subset U M \backslash D$ is an open subset and $p_{1} \in U$. Let $n_{1}=\operatorname{deg} p_{1}$. Then there exists a vector $p_{2} \in U$ close enough to $p_{1}$ with $r_{n_{1}}\left(p_{2}\right) \neq 0$ and $\operatorname{deg} p_{2}=n_{2}>n_{1}$. In a similar way, we find a vector $p_{3}$ such that $r_{n_{1}}\left(p_{3}\right) \neq 0, r_{n_{2}}\left(p_{3}\right) \neq 0$ and $\operatorname{deg} p_{3}=n_{3}>n_{2}$ and so on. This leads to a contradiction, since the number of nonzero terms in (5) is uniformly bounded by $N / 2$.

We need the following lemma:

LEMMA. A compact surface with simple geodesics has constant Gauss curvature.

Proof of THE LemMa. As already shown, we can restrict ourselves to surfaces with closed simple geodesics of the period $2 \pi$. Take a vector $p=$ $(x, u) \in D, \operatorname{deg} p=n$ and let $\gamma=\gamma(t)=f\left(\exp _{x}(t u)\right)$ be the geodesic with initial vector $p$. Let $J_{1}(t), J_{2}(t)$ be linearly independent normal Jacobi vector fields along $\gamma$. Since the degree is locally constant in a neighbourhood of $p$ and the functions $\left\{a, e_{k}, f_{k}\right\}$ are differentiable, the fields $J_{1}, J_{2}$ are trigonometric polynomials in $t$. Keeping the notation $J_{\alpha}$ for $d f\left(J_{\alpha}\right)$ we have 


$$
J_{\alpha}(t)=a_{0}^{\alpha}+\sum_{k=1}^{m}\left(a_{k}^{\alpha} \cos (k t)+b_{k}^{\alpha} \sin (k t)\right), \quad \alpha=1,2,
$$

where $1 \leq m \leq n$ and $a_{k}^{\alpha}, b_{k}^{\alpha} \in \boldsymbol{R}^{N}$. Note that the equation $\left\langle J_{1}^{\prime}(t), J_{2}(t)\right\rangle-$ $\left\langle J_{1}(t), J_{2}^{\prime}(t)\right\rangle=$ const still holds with ${ }^{\prime}=d / d t$, the covariant derivative in $R^{N}$.

Introduce the complex parameter $z=e^{i t}$ and rewrite the equations of Jacobi fields in the form

$$
J_{1}(z)=Q_{1}(z) z^{-m}, \quad J_{2}(z)=Q_{2}(z) z^{-m}, \quad \text { for }|z|=1,
$$

where $Q_{1}, Q_{2}$ are complex vector polynomials of degree $2 m$. The vectors $J_{1}(t)$ and $J_{2}(t)$ are pointwise collinear in $\boldsymbol{R}^{N}$, so $J_{1}(z)$ and $J_{2}(z)$ are pointwise collinear in $\boldsymbol{R}^{N}$ on the circle $|z|=1$, and therefore the polynomial vectors $Q_{1}(z), Q_{2}(z)$ are (complex) collinear for all $z \in C$. Then there exists a nonisotropic vector polynomial $V(z), V: C \rightarrow C^{N}$ and scalar polynomials $R_{1}(z), R_{2}(z)$ such that $Q_{\alpha}=$ $R_{\alpha} V$. Note that $R_{1}(z)$ and $R_{2}(z)$ are linearly independant over $C$.

On the unit circle $d / d t=i z d / d z$ and so $\left\langle J_{1}^{\prime}(z), J_{2}(z)\right\rangle-\left\langle J_{1}(z), J_{2}^{\prime}(z)\right\rangle=$ const, where ' is for $d / d z$ and $\langle$,$\rangle is the Euclidean (not Hermitian!) inner$ product in $C^{N}$. This yields the equation $\left(R_{1}^{\prime} R_{2}-R_{1} R_{2}^{\prime}\right)\langle V, V\rangle=$ const.$z^{2 m-1}$ which holds on the unit circle, and therefore all over $C$. Then $R_{1}^{\prime} R_{2}-R_{1} R_{2}^{\prime}=$ $c_{1} z^{l},\langle V, V\rangle=c_{2} z^{2 m-1-l}$ with complex constants $c_{1}, c_{2}$. The former equation still holds if we pass from $R_{1}, R_{2}$ to their nontrivial linear combinations. So, we can take $R_{1}=z^{a}+\cdots+\beta_{1} z^{b}, R_{2}=\beta_{2} z^{c}+\cdots+z^{d}$, where $a<c, b<d, \beta_{1} \beta_{2} \neq 0$ and dots are for terms of degrees $a<k<b$ in $R_{1}$ and $c<k<d$ in $R_{2}$. Then $R_{1}^{\prime} R_{2}-R_{1} R_{2}^{\prime}=\beta_{2}(a-c) z^{a+c-1}+\cdots+\beta_{1}(b-d) z^{b+d-1}$ and so $R_{1}=z^{a}, R_{2}=z^{d}$, $0 \leq a<d$ and $l=a+d-1$.

Hence $J_{1}(t)=\left(A e^{i a t}+B e^{i d t}\right) V\left(e^{i t}\right) e^{-i m t}$ for some complex constants $A, B$ and $\left\|J_{1}(t)\right\|= \pm \sqrt{c_{2}}\left(A e^{i(a-d) t / 2}+B e^{i(d-a) t / 2}\right)$. This must be real for $t \in[0,2 \pi)$, and therefore $\left\|J_{1}(t)\right\|=A_{1} \cos ((a-d) t / 2)+B_{1} \sin ((a-d) t / 2)$ with $A_{1}, B_{1}$ real constants. So, the Gauss curvature of $M^{2}$ is constant along the geodesic $\gamma$, that is, along any geodesic with initial vector from the open dence subset $D \subset U M$. By continuity, it is constant everywhere on $M^{2}$.

Back to the proof of the Theorem, we conclude that the surface $F^{2}$ is isometric either to a sphere or to a projective plane. However, it will be more convenient to consider $F^{2}$ as the image of a unit sphere under a homothetic immersion $f: S^{2}(1) \rightarrow \boldsymbol{R}^{N}$.

The functions $r_{k}, k=1, \ldots, n$, the amplitudes, initially introduced as the functions on the unit tangent bundle, can be now pulled down on the projective 
plane, the space of geodesics of the sphere, and then lifted up to the sphere. For a point $z \in S^{2}(1) \subset \boldsymbol{R}^{3}$ let $c(t) \subset S^{2}(1)$ be its dual great circle, $t \in[0,2 \pi)$ an arclength parameter. Then

$$
r_{k}(z)=\left(\left(\int_{0}^{2 \pi} f(c(t)) \cos (k t) d t\right)^{2}+\left(\int_{0}^{2 \pi} f(c(t)) \sin (k t) d t\right)^{2}\right)^{1 / 2} .
$$

The functions $r_{k}(z), k=1, \ldots, n$ so defined are even continuous functions on the unit sphere. Making use of (5), we obtain

$$
\|f(x)-f(y)\|^{2}=\sum_{k=1}^{n} r_{k}^{2}(z)(2-2 \cos (k d(x, y))),
$$

where $x, y \neq \pm x \in S^{2}(1), z$ is a unit vector orthogonal both to $x$ and $y$, and $d(x, y)$ is the spherical distance.

An isometric (or a homothetic) immersion of a compact Riemannian manifold in a Euclidean space is called an immersion of finite type, if all the components of its position vector are finite sums of eigenfunctions of the Laplacian on the manifold [3]. In particular, an immersion of a circle given by (5) is of finite type. Then, from [4, Théorème 1], the surface $F^{2}$ is a finite type immersed sphere. Since the eigenvectors of the Laplacian of $S^{2}$ are homogeneous polynomials in $\boldsymbol{R}^{3}$ restricted to $S^{2}$, we conclude that $F^{2}$ is the image of $S^{2}(1) \subset \boldsymbol{R}^{3}$ under a polynomial map $F: \boldsymbol{R}^{3} \rightarrow \boldsymbol{R}^{N}$.

Show that $F$ is an SG-map as in Definition 3. First of all, prove that it splits on the orthogonal sum of an even and an odd map. Let $F=F_{1}+F_{2}$, where $F_{1}$ is an even polynomial map and $F_{2}$ is an odd one. Since we are interested only in the values of the map $F$ on the unit sphere, we can multiply the terms of lower degree by an appropriate power of $\|x\|^{2}$ to make $F_{1}$ and $F_{2}$ homogeneous. Moreover, after parallel translation we may assume $F$ to be mass-symmetric, that is, $\int_{S^{2}} F(x) d A(x)=0$. Let $x$ and $y$ be two points on $S^{2}(1)$ on the distance $0<t_{0}<\pi$ and let $z \in \boldsymbol{R}^{3}$ be a unit vector collinear to $x \times y$. From (6)

$$
\begin{aligned}
\|F(x)-F(y)\|^{2}= & \sum_{k=1}^{n} r_{k}^{2}(z)\left(2-2 \cos \left(k t_{0}\right)\right) \\
= & \left\|F_{1}(x)-F_{1}(y)\right\|^{2}+\left\|F_{2}(x)-F_{2}(y)\right\|^{2} \\
& +2\left\langle F_{1}(x)-F_{1}(y), F_{2}(x)-F_{2}(y)\right\rangle .
\end{aligned}
$$

Replacing $(x, y)$ by $(-x,-y)$ we get $\left\langle F_{1}(x)-F_{1}(y), F_{2}(x)-F_{2}(y)\right\rangle \equiv 0$. Averaging this equation over the unit sphere $\{\|y\|=1\}$ we obtain $\left\langle F_{1}(x), F_{2}(x)\right\rangle \equiv 0$; 
and therefore $\left\langle F_{1}(x), F_{2}(y)\right\rangle+\left\langle F_{1}(y), F_{2}(x)\right\rangle \equiv 0$. Finally, changing $y$ to $-y$ we get $\left\langle F_{1}(x), F_{2}(y)\right\rangle \equiv 0$ for all $x, y \in S^{2}(1) \subset \boldsymbol{R}^{3}$. This gives an orthogonal decomposition $\boldsymbol{R}^{N}=\boldsymbol{R}^{N_{1}} \oplus \boldsymbol{R}^{N_{2}}$ such that $F_{1}: \boldsymbol{R}^{3} \rightarrow \boldsymbol{R}^{N_{1}}$ and $F_{2}: \boldsymbol{R}^{3} \rightarrow \boldsymbol{R}^{N_{2}}$.

We shall write $F_{e}, F_{o}$ instead of $F_{1}, F_{2}$ respectively. Both $F_{e}$ and $F_{o}$ (if nonzero) map great circles of the unit sphere $S^{2}(1) \in \boldsymbol{R}^{3}$ onto $W$-curves. We want to show that the maps $F_{e}, F_{o}$ satisfy (3), (4) respectively.

Consider the map $F_{o}$ first. Since $F_{o}$ is odd, it maps the great circles on $W$-curves with odd frequences only, that is, on the curves of the form

$$
\gamma(t)=\sum_{k=1}^{l}\left(e_{2 k-1} \cos ((2 k-1) t)+f_{2 k-1} \sin ((2 k-1) t)\right),
$$

where $2 l-1=\operatorname{deg} F_{o}$ and $\left\{e_{2 j-1}, f_{2 k-1}\right\}, j, k=1, \ldots, l$ are orthogonal vectors in $\boldsymbol{R}^{N_{1}}$, not all zeros, with $\left\|e_{2 k-1}\right\|=\left\|f_{2 k-1}\right\|$. Then $\|\gamma(t)\|$ is constant and therefore $\left\|F_{o}(x)\right\|_{\mid S^{2}(1)}$ is constant, hence $F_{o}\left(S^{2}(1)\right)$ lies in a sphere in $\boldsymbol{R}^{N_{1}}$ centered at the origin. Furthermore, from (6) for orthonormal vectors $x, z \in \boldsymbol{R}^{3}$

$$
\left\langle F_{o}(x), F_{o}(\cos t x+\sin t x \times z)\right\rangle=\sum_{k=1}^{l} r_{2 k-1}^{2}(z) \cos ((2 k-1) t) .
$$

Decomposing the left-hand side by sines and cosines of multiple angles and taking into account the parity arguments, we see that $r_{2 k-1}^{2}(z), k=1, \ldots, l$ are polynomials in $x, z$ which are of even degree both in $x$ and in $z$. Integrating by $x$ over a unit circle in $S^{2}(1)$ dual to $z$ we find that every function $r_{2 k-1}^{2}$ is a restriction of an even polynomial to $S^{2}(1)$.

From (6), for unit vectors $x, y \neq \pm x$

$$
\left\langle F_{o}(x), F_{o}(y)\right\rangle=\sum_{k=1}^{l} r_{2 k-1}^{2}\left(\frac{x \times y}{\|x \times y\|}\right) \cos ((2 k-1) d(x, y)) .
$$

Decomposing $\cos ((2 k-1) d(x, y))$ by odd powers of $\langle x, y\rangle$ and substituting $r_{2 k-1}^{2}$, we get

$$
\left\langle F_{o}(x), F_{o}(y)\right\rangle=\sum_{k=1}^{l}\langle x, y\rangle^{2 k-1} S_{2 l-2 k}(x \times y)\|x \times y\|^{-2 p},
$$

where $S_{2 l-2 k}, k=1, \ldots, l$ are even polynomials in $\boldsymbol{R}^{3}$. Since $F_{o}$ is homogeneous and $\|x\|^{2}\|y\|^{2}=\|x \times y\|^{2}+\langle x, y\rangle^{2}$, this equation remains valid for any pair of non-collinear vectors $x, y \in \boldsymbol{R}^{3}$ (with some different even polynomials $S_{2 l-2 k}$ ). We claim that all the $\left\{S_{2 l-2 k}(z)\right\}$ are divisible by $\|z\|^{2 p}$. Indeed, suppose that for some $1 \leq n \leq p$ all the polynomials $\left\{S_{2 l-2 k}(z)\right\}$ are divisible by $\|z\|^{2 p-2 n}$, but at least 
one of them is not divisible by $\|z\|^{2 p-2 n+2}$. Multiplying both sides of the latter equation by $\|x \times y\|^{2 n}$ we get

$$
\|x \times y\|^{2} Q(x, y)=\sum_{i}\langle x, y\rangle^{2 k_{i}-1} T_{i}(x \times y),
$$

where none of the even polynomials $T_{i}=T_{i}(z)$ is divisible by $\|z\|^{2}$. This polynomial equation holds for any non-collinear vectors $x, y \in \boldsymbol{R}^{3}$, and therefore, for any $x, y \in C^{3}$. Then by the Hilbert zero theorem there exists $z_{0} \in C^{3}$ such that $\left\|z_{0}\right\|^{2}=0$ and $T_{i}\left(z_{0}\right) \neq 0$ for all $i$. Choose $x_{0}, y_{0} \in C^{3}, x_{0}$ nonisotropic, such that $x_{0} \times y_{0}=z_{0}$. Then the substitution of $x=x_{0}, y=t x_{0}+y_{0}, t \in \boldsymbol{C}$ to (7) leads to a contradiction.

Thus, the equation

$$
\left\langle F_{o}(x), F_{o}(y)\right\rangle=\sum_{k=1}^{l}\langle x, y\rangle^{2 k-1} S_{2 l-2 k}(x \times y),
$$

holds for all $x, y \in \boldsymbol{R}^{3}$ with $S_{2 l-2 k}, k=1, \ldots, l$ even polynomials in $\boldsymbol{R}^{3}$. Comparing the degrees, we see that $\left\{S_{2 l-2 k}\right\}$ are homogeneous and $\operatorname{deg} S_{2 l-2 k}=$ $2 l-2 k$. So, the map $F_{o}$ satisfies (4).

The arguments for the even map $F_{e}: \boldsymbol{R}^{3} \rightarrow \boldsymbol{R}^{N_{2}}$ are pretty much the same as for $F_{o}$, although the image $F_{e}\left(S^{2}(1)\right)$ does not need to lie in a sphere in $\boldsymbol{R}^{N_{2}}$. By (6)

$$
\left\|F_{e}(x)-F_{e}(\cos t x+\sin t x \times z)\right\|^{2}=4 \sum_{k=1}^{m} r_{2 k}^{2}(z) \sin ^{2}(k t) .
$$

for any orthonormal vectors $x, z \in \boldsymbol{R}^{3}$. Then every function $r_{2 k}^{2}$ is a restriction of an even polynomial in $\boldsymbol{R}^{3}$ to $S^{2}(1) \subset \boldsymbol{R}^{3}$.

Using (6) again, with unit vectors $x, y \neq \pm x \in \boldsymbol{R}^{3}$, we get

$$
\left\|F_{e}(x)-F_{e}(y)\right\|^{2}=P(x \times y)\|x \times y\|^{-2 p}
$$

for some even polynomial $P$. To establish (3) we need to prove that the righthand side is actually a polynomial, that is, that $P(z)$ is divisible by $\|z\|^{2 p}$. Suppose this is not true and the right-hand side of the latter equation is already reduced to the least possible value of $p$. Then the polynomial $P(z)$ is not divisible by $\|z\|^{2}$ and with some polynomial $Q$

$$
\|x \times y\|^{2} Q(x, y)=P(x \times y)
$$


on the product of unit spheres in $\boldsymbol{R}^{6}$. This equation still holds on the complex quadric $\left\{(x, y) \in \boldsymbol{C}^{6}: x_{1}^{2}+x_{2}^{2}+x_{3}^{2}=y_{1}^{2}+y_{2}^{2}+y_{3}^{2}=1\right\}$. Indeed, let $R(x, y)$, $x \in \boldsymbol{R}^{3}, y \in \boldsymbol{R}^{3}$ be a real polynomial that vanishes on the product of unit spheres $\{\|x\|=\|y\|=1\}$ in $\boldsymbol{R}^{3} \oplus \boldsymbol{R}^{3}=\boldsymbol{R}^{6}$. Perform an inversion with the center $(1,0,0)$ and the radius 2 in the $x$-space, and with the center $(1,0,0)$ and the radius 2 in the $y$-space. The polynomial $R$ then transforms to a rational function $\tilde{R}(x, y)$ vanishing on the linear subspace $x_{1}=y_{1}=0$. Then the complex rational function $\tilde{R}(x, y), x, y \in C^{3}$ vanishes on the subspace $x_{1}=y_{1}=0$ in $C^{6}$ and so does $R(x, y)$ on the complex quadric $\left\{(x, y) \in C^{6}:\|x\|^{2}=\|y\|^{2}=1\right\}$.

By the Hilbert zero theorem we can find an isotropic nonzero vector $z_{0} \in \boldsymbol{C}^{3}$ such that $P\left(z_{0}\right) \neq 0$. Let $z_{0}=u+i v, u, v \in \boldsymbol{R}^{3}$ and $w \in \boldsymbol{R}^{3}$ a unit vector orthogonal both to $u$ and $v$ and such that the triple $(u, v, w)$ is positively oriented in $\boldsymbol{R}^{3}$. Then the cross-product of the unit vectors $x=w-i z_{0}$ and $y=w$ is $z_{0}$, which leads to a contradiction in (8).

Thus, the polynomial map $F_{e}$ satisfies (3) and, consequently, the map $F$ is a simple geodesic.

The author would like to thank the referee for valuable comments.

\section{References}

[1] Besse, A., Manifolds all off whose geodesics are closed, Springer-Verlag, 1978.

[2] Bieberbach, L., Eine singularitätenfreie Fläche konstanter negative Krümmung in Hilbertschen Räume, Comment. Math. Helv. 4 (1932), 248-255.

[ 3 ] Chen, B.-Y., A report on submanifolds of finite type, Soochow J. Math. 22 (1996), 117-337.

[4] Chen, B.-Y., Deprez, J., Verheyen, P., Immersions, dans un espace euclidien, d'un espace symétrique compact de rang un à géodésiques simples, C. R. Acad. Sci. Paris Sér. I Math. 304 (1987), 567-570.

[ 5] Damek, E., Ricci, F., A class of nonsymmetric harmonic Riemannian spaces, Bull. Amer. Math. Soc. 27 (1992), 139-142.

[6] Ferus, D., Schirrmacher, S., Submanifolds in Euclidean space with simple geodesics, Math. Ann. 260 (1982), 57-62.

[ 7] Helgason, S., Topics in harmonic analysis on homogeneous spaces, vol. 13, Progress in Mathematics, Birkhäuser, Boston, 1981.

[ 8 ] Kim, Y. H., Surfaces with simple geodesics, J. Korean Math. 30 (1993).

[9] Klein, F., Lie, S., Über diejenigen ebenen Kurven, welche durch ein geschlossenes System von einfach unendlich vielen vertauschbaren linearen Transformationen in sich übergehen, Math. Ann. 4 (1871), 50-84.

[10] Mutō, Y., Geodesics in minimal immersions of $S^{3}$ into $S^{24}$., Tokyo J. Math. 13 (1990), 221234.

[11] Sakamoto, K., Helical immersions into a unit sphere, Math. Ann. 261 (1982), 63-80.

[12] Szabo, Z. I., The Lichnerowicz conjecture on harmonic manifolds, J. Diff. Geom. 31 (1990), $1-$ 28.

[13] Tsukada, K., Helical geodesic immersions of compact rank one symmetric spaces into spheres, Tokyo J. Math. 6 (1983), 267-285. 
Department of Mathematics

La Trobe University, Bundoora, 3083

Victoria, Australia

E-mail address: Y.Nikolayevsky@latrobe.edu.au 